\title{
Feasibility of TEE-guided stroke risk assessment in atrial fibrillation-background, aims, design and baseline data of the TIARA pilot study
}

\author{
T. Dinh • L. H. B. Baur • R. Pisters • O. Kamp • \\ F. W. A. Verheugt • J. L. R. M. Smeets • E. C. Cheriex • \\ R. G. Tieleman • M. H. Prins • H. J. G. M. Crijns • \\ for the TIARA pilot study group
}

Published online: 23 March 2011

(C) The Author(s) 2011. This article is published with open access at Springerlink.com

\begin{abstract}
Background Antithrombotic management in atrial fibrillation (AF) is currently based on clinical characteristics, despite evidence of potential fine-tuning with transoesophageal echocardiography (TEE). This open, randomised, multicentre study addresses the hypothesis that a comprehensive strategy of TEE-based aspirin treatment in AF patients is feasible and safe.
\end{abstract}

The participants in the TIARA pilot study are listed in the Appendix

T. Dinh $(\varangle) \cdot$ R. Pisters $\cdot$ E. C. Cheriex $\cdot$ H. J. G. M. Crijns

Department of Cardiology, Maastricht University Medical Center, PO Box 5800, 6202 AZ, Maastricht, the Netherlands

e-mail: trang.dinh@mumc.nl

\section{H. B. Baur}

Department of Cardiology, Atrium Medical Center,

Heerlen, the Netherlands

O. Kamp

Department of Cardiology, VU University Medical Center,

Amsterdam, the Netherlands

F. W. A. Verheugt

Department of Cardiology, Onze Lieve Vrouwe Gasthuis,

Amsterdam, the Netherlands

\section{J. L. R. M. Smeets}

Department of Cardiology,

Radboud University Nijmegen Medical Center,

Nijmegen, the Netherlands

R. G. Tieleman

Department of Cardiology, Martini Hospital Groningen,

Groningen, the Netherlands

M. H. Prins

Department of Clinical Epidemiology, Maastricht University Medical Center,

Maastricht, the Netherlands
Methods Between 2005 and 2009, ten large hospitals in the Netherlands enrolled AF patients with a moderate risk of stroke. Patients without thrombogenic TEE characteristics were randomised to aspirin or vitamin $\mathrm{K}$ antagonists (VKA). The primary objective is to show that TEE-based aspirin treatment is safe compared with VKA therapy. The secondary objective tests feasibility of TEE as a tool to detect echocardiographic features of high stroke risk. This report compares randomised to non-randomised patients and describes the feasibility of a TEE-based approach. Results In total, 310 patients were included. Sixty-nine patients were not randomised because of non-visualisation $(n=6)$ or TEE risk factors $(n=63)$. Compared with nonrandomised patients, randomised patients $(n=241)$ were younger ( $65 \pm 11$ vs. $69 \pm 9$ years, $p=0.004)$, had less coronary artery disease ( 9 vs. $20 \%, p=0.018$ ), previous TIA ( 1.7 vs. $7.2 \%, p=0.029$ ), AF during TEE ( 25 vs. $54 \%$, $p<0.001$ ), mitral incompetence (55 vs. $70 \%, p=0.038$ ), VKA use (69 vs. $82 \%, p=0.032$ ), had a lower mean $\mathrm{CHADS}_{2}$ score $(1.2 \pm 0.6$ vs. $1.6 \pm 1.0, p=0.004)$, and left ventricular ejection fraction ( $59 \pm 8$ vs. $56 \pm 8 \%, p=0.016$ ).

Conclusions This study shows that a TEE-based approach for fine-tuning stroke risk in $\mathrm{AF}$ patients with a moderate risk for stroke is feasible. Follow-up data will address the safety of this TEE-based approach.

Keywords Atrial fibrillation - Stroke risk · Antithrombotic treatment

\section{Introduction}

Atrial fibrillation (AF) is an independent risk factor for stroke, and patients with $\mathrm{AF}$ have a 5\% annual risk for thromboem- 
bolic stroke, i.e. five times more than in patients without AF $[1,2]$. Therapy with vitamin $\mathrm{K}$ antagonists (VKA) reduces the risk of thromboembolism dramatically: ischaemic stroke by $67 \%$ and peripheral thromboembolism by $42 \%$ [3]. Alternatively, aspirin reduces the risk of ischaemic stroke by $22 \%$, but has a significantly lower risk of bleeding [4].

It is recommended to stratify the risk of ischaemic stroke in patients with AF using straightforward clinical characteristics [5]. However, many AF patients classified as having a high or moderate risk for thromboembolism may actually be at low risk, as was shown in the Stroke Prevention in Atrial Fibrillation (SPAF) III study. In SPAF III, a subset of patients with a very high risk of stroke underwent transoesophageal echocardiography (TEE) [6]. Complex plaques in the aorta and signs of atrial stasis (spontaneous echo contrast, low peak emptying velocities and presence of thrombus in the left atrium or left atrial appendage) appeared strongly associated to a high risk of ischaemic stroke and peripheral thromboeobolism [6-8]. Conversely, patients with a normal TEE had an extremely low rate of thromboembolism despite the fact that they had just used aspirin. Up till now no prospective study on the clinical safety and feasibility of a TEE-based risk assessment has been performed.

In the TEE-guIded randomized comparison of AspiRin and VKA in patients with AF and an increased risk of stroke (TIARA) pilot study we hypothesise that a comprehensive strategy of TEE-based aspirin treatment is safe and feasible in AF patients who are eligible for vitamin $\mathrm{K}$ antagonist therapy on the basis of conventional risk assessment. Our primary objective is to show thatcompared with VKA therapy-TEE-based aspirin treatment is safe with respect to a composite endpoint of major cardiovascular and cerebrovascular events, in patients who do not harbour predefined high-risk features on TEE. Our secondary objective is to test the feasibility of TEE as a tool to assess all four echocardiographic features of high stroke risk, i.e. complex atheromatous plaques in the thoracic aorta and signs of atrial stasis including dense spontaneous echo contrast, thrombus or low blood flow velocity.

Here we report the study design and feasibility of TEEguided stroke risk assessment. In addition we compare the baseline characteristics of randomised patients with those of patients who were not randomised because of thrombogenic TEE abnormalities.

\section{Methods}

\section{Patients}

Subjects were eligible for participation if they had documented paroxysmal or permanent AF, and a conventional indication for VKA treatment (history of hypertension, age $>60$ years with diabetes mellitus or coronary artery disease, age $>75$ years). Subjects were excluded if they had an indication for VKA treatment other than AF, secondary AF, a contraindication for treatment with VKA or aspirin or a contraindication for TEE [9]. Also patients with a presumed very high risk of stroke and thromboembolism (previous ischaemic stroke, transient ischaemic attack (TIA) or systemic thromboembolism, heart failure or significant left ventricular systolic dysfunction, mitral valve stenosis, hypertrophic cardiomyopathy) were not eligible. The Institutional Review Boards of all participating hospitals approved the study and written informed consent was obtained from all patients. Since the risk of stroke in patients who suffered from TIA but not from stroke is uncertain and its mechanisms vary, the steering committee changed the exclusion criteria of the study accepting also patients with a history of TIA for screening. This protocol amendment was implemented in March 2006.

\section{Design}

The TIARA pilot study was an open randomised, multicentre trial in which a risk stratification based on TEE was examined in AF patients with a moderate risk of stroke. The study was performed in ten large hospitals in the Netherlands between 2005 and 2009. All centers are highly experienced in echocardiography. Most patients were enrolled in the outpatient clinic, but also hospitalised patients were included. The trial has been registered under ClinicalTrials.gov \# NTC 00224757.

Patients underwent a TEE and were randomly assigned to aspirin or VKA treatment if TEE did not show high-risk abnormalities. Patients assigned to aspirin received a oncedaily dose of $100 \mathrm{mg}$ and patients assigned to VKA received adjusted-dose acenocoumarol or phenprocoumon (target INR 2.5 to 3.5). Obviously, patients with one or more abnormalities on TEE were excluded from randomisation and received adjusted-dose VKA and were followed in the high-risk observational group. The Netherlands' regional Thrombosis Service routinely checked the INR. All patients were followed up for at least 1 year. Figure 1 depicts the flow chart of screening, randomisation and follow-up of included patients in the TIARA pilot study.

Echocardiographic procedures

All TEEs were performed and interpreted by certified cardiologists in a standard fashion, as recommended by the American Society of Echocardiography [9]. All echocardiograms were acquired by commercially available equipment: HP Sonos 5500 imaging system (Hewlett-Packard Co., Andover, MA) or Vingmed System V (General Electric Company, Fairfield, Connecticut) equipped with a $5-\mathrm{MHz}$ omniplane probe for TEE. When in doubt, the echocardiograms 


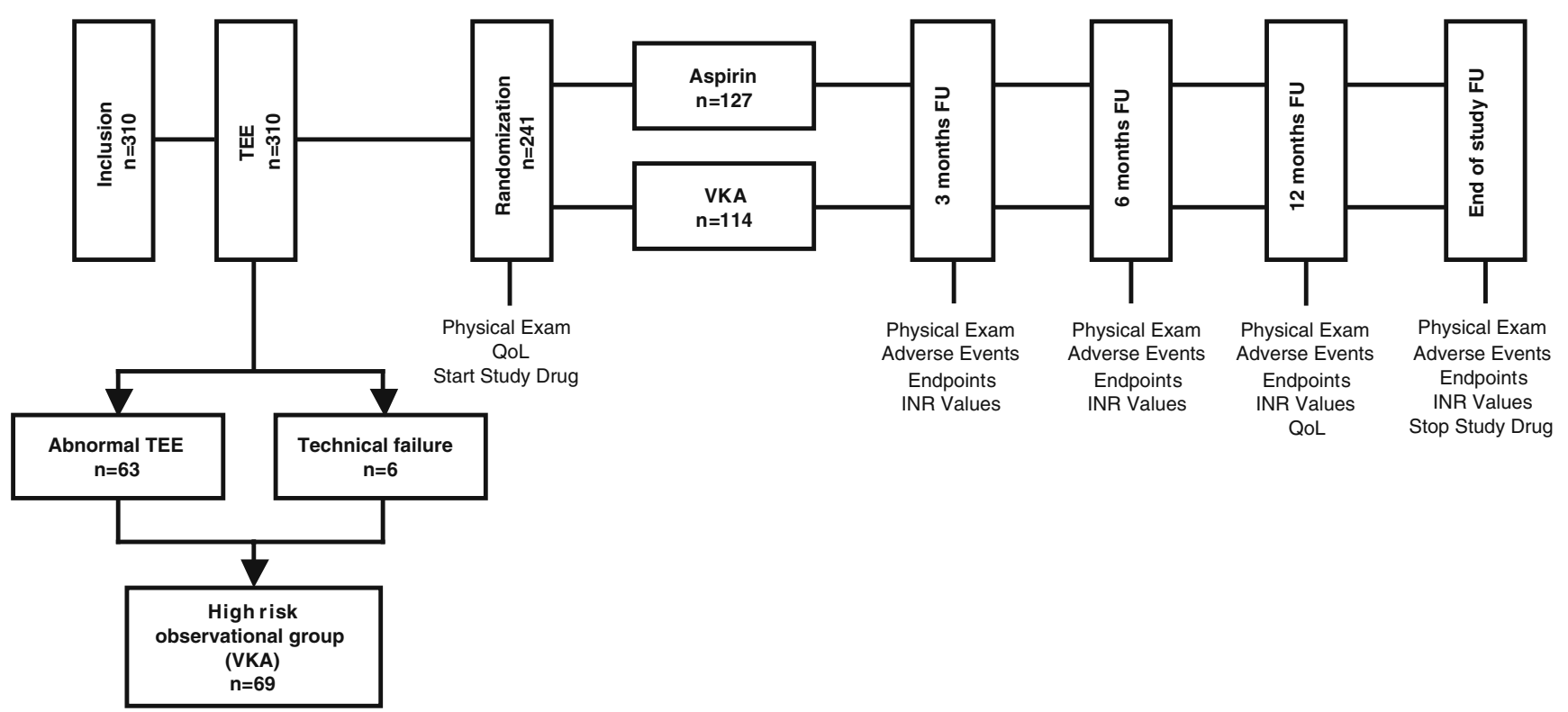

Fig. 1 Overview of the screening, randomisation and follow-up of the TIARA pilot study. Eligible patients were planned for TEE to assess presence or absence or echocardiographic features of high risk of stroke i.e. signs of atrial stasis and complex aortic plaques. VKA vitamin $\mathrm{K}$ antagonists, TEE transoesophageal echocardiography,

could be sent to the echo core laboratory (Maastricht University Medical Center) for review.

TEE was performed with a 5-MHz omniplane transducer. The oropharynx was anaesthetised by use of topical lidocaine spray and viscous lidocaine solution prior to insertion of the probe. The left atrial appendage (LAA) was imaged by placing the probe at the level of the midoesophagus. The plane of the multiplane probe was adjusted to achieve a short-axis view of the aortic valve $\left(45^{\circ}\right)$; to obtain an optimal view of the left atrial appendage, the shaft of the scope was rotated anticlockwise in 5 to $10^{\circ}$ increments from 0 to $180^{\circ}$. Multiple views of the LAA were then obtained; cine loops were acquired [10]. The imaging plane and gain settings were adjusted for optimal visualisation of thrombi and spontaneous echo contrast (SEC) in the left atrium (LA), LAA, right atrium (RA) and right atrial appendage (RAA). For measurement of the blood flow velocities in the LAA, the sample of the pulsed wave Doppler was placed at the orifice of the LAA and the profile of the velocities was recorded over at least five cardiac cycles. A maximum velocity was then calculated by averaging these five cardiac cycles [11]. In order to image complex plaques, the thoracic aorta was carefully visualised by retracting and rotating the probe.

Definition of high-risk echocardiographic features

The following definitions were used for echocardiographic abnormalities. Spontaneous echo contrast was defined as a
Positive TEE presence of at least one of the echocardiographic features of high risk of stroke, Negative TEE absence of echocardiographic features of high risk of stroke, QoL Quality of life scores using SF-36, EuroQol 5-D, Minnesota and AFSS questionnaires

persistent pattern of a slow swirling motion of intracavitary echo density throughout the LA and LAA at normal gain [6]. A thrombus was defined as an intracavitary echodense mass with sharp edges, echogenicity different from adjacent structures, some degree of mobility, and present in more than one plane [12]. Low blood flow velocity was defined as peak emptying velocity of less than $25 \mathrm{~cm} / \mathrm{s}$ on TEE, measured at the orifice of the LAA. Complex atheromatous plaque was defined as a mobile, pedunculated or ulcerated plaque, or plaque thickness $\geq 4 \mathrm{~mm}$ in the thoracic aorta [7].

\section{Randomisation}

Randomisation was performed by permuted block design with stratification for each participating hospital. Eligible patients were randomised to either treatment with aspirin $100 \mathrm{mg}$ once daily or treatment with adjusteddose VKA. In patients randomised to VKA, the INR was checked by the regional Thrombosis Service on a routine basis.

\section{Follow-up}

Patients visited the outpatient clinic at 3,6 and 12 months after randomisation and at the end of the study. All patients were followed up for at least 1 year. If needed, patients were seen between regular visits. At every visit, symptoms and signs of the composites of the primary endpoint were checked. Also INR values were collected. 
TEE was not repeated on a regular basis in the included patients. Within the study period it was not expected that aortic plaque or left atrial low flow states would develop if not present on the first examination. A TEE was, however, repeated when clinically indicated. If electrical cardioversion was indicated, aspirin patients were temporarily switched to VKA. One month after the shock, irrespective of the rhythm, aspirin was reinstituted.

\section{Quality of life questionnaires}

The quality of life was studied using the EuroQol 6-D, the Short Form (SF)-36 Health Survey questionnaire and the Toronto Atrial Fibrillation Severity Scale (AFSS) at inclusion and after 12 months of follow-up. SF-36 contained items to assess physical health and mental health, AFSS secured AF-specific complaints. The quality of life assessment was completed with a multidimensional index that contains five subscales: general, physical, activity, motivation and cognition.

\section{Data collection}

Data were collected using an electronic case report form accessed at a secure Internet site. Data were transferred through the Internet to the central database in Maastricht. Data could be either entered online, or offline with frequent data transfer. By using a validation plan, integrated in the data entry software, data were continuously checked for missing or contradictory entries and values out of the normal range. The studymonitoring nurse performed additional edit checks. The data analysis was done at the Maastricht University Medical Center. Patient identification was registered in the participating centres, and was not transferred to the central database. Questionnaires for quality of life were processed by the Center for Data and Information Management (MEMIC), a subdivision of the department of the Faculty of Health, Medicine and Life Sciences (Maastricht University).

\section{Endpoints}

The primary endpoint was a composite of (1) stroke including haemorrhagic strokes, (2) systemic embolism, (3) major bleeding, (4) acute coronary syndrome (unstable angina and myocardial infarction) and (5) all-cause mortality.

The Central Independent Adjudication Committee (CIAC) independently adjudicated all events using definitions as mentioned below. In case of doubt the members held a conference for an unanimous adjudi- cation. Definitions of primary endpoints are described in the Appendix.

\section{Statistical analysis}

Sample size calculation was driven by the primary endpoint on safety, i.e. the composite endpoint. The incidence of the composite safety endpoint with aspirin treatment could be estimated from the available literature. In patients with a normal TEE, the estimated yearly incidences of ischaemic stroke and systemic thromboembolism, major bleeding, acute coronary syndrome and death with aspirin treatment were $1.1 \%$, $0.7 \%, 0.8 \%$ and $1.8 \%$, respectively. Therefore, the estimated yearly incidence of the composite primary endpoint was $4.4 \%$ with aspirin. With VKA treatment (INR 2.0-3.0) the yearly incidences of ischaemic stroke and systemic thromboembolism, major bleeding, acute coronary syndrome and death were $1.0 \%, 1.7 \%, 1.0 \%$ and $1.8 \%$, respectively. As a result, the estimated yearly incidence of the composite endpoint with VKA treatment was 5.5\%. With an estimated average follow-up duration of 1.5 years, the estimated incidences of the composite safety endpoint were $6.6 \%$ for the aspirin group and $8.3 \%$ for the VKA group, respectively.

The primary safety objective of this pilot study is to show that applying TEE-based aspirin prophylaxis does not lead to unacceptable high frequency of the safety endpoint. Inclusion of 300 patients (150 per randomisation arm) would rule out an excess of $7 \%$ with a power of $80 \%$ and a type I error of $5 \%$. The difference in incidence of the safety endpoint in the randomised groups was calculated and described with an exact onesided 95\% confidence interval. A Kaplan-Meier curve will be used to visualise the occurrence of events over time. Hazard ratios will be calculated using Cox regression. Similar analyses will be done for the separate components of the safety endpoint.

The secondary - feasibility_objective will be evaluated by calculating the number of patients eligible for the TEE approach in whom the TEE cannot be performed or does not yield unequivocal information on echocardiographic thromboembolism risk markers.

Data that are not normally distributed will be logtransformed and presented as geometric means. Analyses were done using the $t$-test for independent groups for continuous variables and Fisher's exact test for categorical variables. SPSS was used for all statistical calculations. Analyses will be done using the intention-to-treat principle, that is, patients will be analysed in the group to which they were randomly assigned, regardless of the treatment they actually received. A P value less than 0.05 is considered statistically significant. All tests are two-tailed. 


\section{Results}

\section{Baseline characteristics}

The inclusion in the TIARA pilot trial was slower than expected and the follow-up period longer than foreseen. As a consequence inclusion was stopped after 310 patients and a projected mean follow-up duration of 1.6 years. Recruitment was diminished predominantly due to refusal of eligible patients to undergo a TEE examination because of fear for the TEE. Analysis of the patient screening log showed that $35 \%$ of patients eligible for participation in the TIARA pilot study refused TEE for this reason.

In the group randomised to aspirin, three patients withdrew their informed consent directly after randomisation. Therefore they will be discarded from further analysis in respect to follow-up. For the secondary (per protocol) analysis, patients not meeting the inclusion $(n=1$ for VKA group, $n=2$ for aspirin group), or exclusion criteria ( $n=3$ for VKA group), and patients randomised despite TEE abnormalities $(n=1$ for VKA group), will be discarded from analysis.

We had included 310 patients by July 2008, of which $241(78 \%)$ patients were randomised. In total, 69 patients were not randomised on the basis of an abnormal TEE showing either predefined echocardiographic markers of a high stroke risk or another abnormality considered highly thrombogenic. Summaries of clinical and echocardiographic data are expressed as means or frequencies with $95 \%$ confidence intervals (CIs) (Table 1). Non-randomised observational patients had a slightly higher $\mathrm{CHADS}_{2}$ score due to on average higher age, higher prevalence of diabetes mellitus and previous TIA. The prevalence of hypertension was very high and similar in both groups. On the other hand, the prevalence of TIA was low, while patients with stroke and heart failure were excluded. Non-randomised observational patients were more often in AF at the time of TEE and had mitral incompetence of any grade more frequently. They also more often used a vitamin $\mathrm{K}$ antagonist at entry. Distribution of type of AF (paroxysmal or permanent) did not differ among groups.

\section{Feasibility of TEE-guided stroke risk assessment}

Of all 310 patients planned for TEE, 69 were not eligible for randomisation because of non-visualisation $(n=5)$, technical failure $(n=1)$ or abnormal TEE $(n=63)$ (Table 2$)$. At least one echocardiographic marker for thromboembolism could not be visualised with certainty in five patients; mostly due to incomplete imaging of the LAA and therefore LAA flow velocities could not be measured $(n=4)$. In the remaining patient, the aorta could not be visualised. In one patient, TEE could not be performed because of technical reasons; the patient was not capable of swallowing the probe and therefore none of the markers for thromboembolism were visualised. Furthermore, 63 patients had abnormalities on TEE, of which 60 patients $(19.7 \%)$ had at least one predefined risk marker on TEE. Thrombi were detected in ten patients (3.3\%), low LAA flow velocities were seen in 33 patients $(10.9 \%)$, spontaneous echo contrast in 22 patients $(7.2 \%)$, and complex plaques in 13 patients $(4.3 \%)$. The majority had only one risk marker $(n=49$, Table 2$)$; LAA flow velocities, SEC, complex plaques and thrombi were seen in 20, 11, 9 and 6 patients, respectively. In 13 patients, two abnormalities were detected with TEE; seven patients had low LAA flow velocities with SEC, three patients had a thrombus with low LAA flow velocities, and three patients had SEC with complex plaques. Only one patient had all four markers of thromboembolism risk on TEE.

Surprisingly, other cardiac abnormalities were observed in three patients who had no other risk markers for stroke; two patients had Lambl's excrescences on the aortic valve and one patient had a myxoma in the left atrium. Lambl's excrescences are not considered to be a primary source of cardioembolism in patients with sinus rhythm, but the importance in atrial fibrillation remains uncertain [13]. These patients were therefore not randomised. The patient with a myxoma refused surgery, and therefore was not randomised because of the need for permanent anticoagulation.

Thus, TEE could provide accurate information on thromboembolic risk in 304 out of 310 patients (98.1\%).

\section{Discussion}

The TIARA pilot study is a randomised comparison of aspirin and vitamin $\mathrm{K}$ antagonists for stroke prevention in patients with a moderate stroke risk who do not harbour predefined high-risk features on TEE. It was constructed as a pilot study to show feasibility and to prepare a larger study later on. Considering the high number of equivocal TEE imaging results (98.1\% of patients) and the low number of technical failures of TEE, we feel that a TEE-guided approach is feasible in clinical practice. If our study shows that TEE-based aspirin treatment is also safe, this may form the basis for a large TEE-guided stroke risk assessment trial.

We found that in only six out of the 310 included patients $(1.9 \%)$ irrefutable information about the thrombogenicity could not be obtained by TEE. In one patient a TEE could not be performed due to technical reasons, and in the remaining five patients the left atrial appendage and aorta could not be visualised accurately. This high technical success rate may relate to the fact that all participating centers in the TIARA pilot study were highly trained in performing TEE. However, TEE is now well incorporated in the daily practice of any cardiology clinic. We hence believe that a TEE-based approach for fine-tuning stroke 
Table 1 Characteristics of randomised and non-randomised patients

\begin{tabular}{|c|c|c|c|}
\hline & Randomised patients & $\begin{array}{l}\text { Non-randomised } \\
\text { patients } \\
\text { (observational group) }\end{array}$ & $p$-value \\
\hline & $n=241$ & $n=69$ & \\
\hline \multicolumn{4}{|l|}{ Clinical characteristics } \\
\hline Age (years) & $65 \pm 11$ & $69 \pm 9$ & 0.004 \\
\hline Male, $\%$ & 66 & 61 & 0.479 \\
\hline Hypertension, \% & 90 & 86 & 0.388 \\
\hline Coronary artery disease, $\%$ & 9 & 20 & 0.018 \\
\hline Diabetes, $\%$ & 10 & 19 & 0.057 \\
\hline Transient ischaemic attack, $\%$ & 1.7 & 7.2 & 0.029 \\
\hline $\begin{array}{l}\mathrm{CHADS}_{2} \text { score } \\
\text { - Score } 0, \%\end{array}$ & $\begin{array}{l}1.2 \pm 0.6 \\
2.1\end{array}$ & $\begin{array}{l}1.6 \pm 1.0 \\
4.3\end{array}$ & $<0.001$ \\
\hline - Score $1, \%$ & 76.3 & 53.6 & \\
\hline - Score $\geq 2, \%$ & 21.6 & 42.1 & \\
\hline Time since 1st diagnosis AF (years) & $4.9 \pm 9.0$ & $4.6 \pm 6.1$ & 0.754 \\
\hline $\begin{array}{l}\text { Type of AF } \\
\text { - Paroxysmal, \% }\end{array}$ & 85 & 78 & 0.320 \\
\hline - Permanent, $\%$ & 15 & 22 & \\
\hline Body mass index $\left(\mathrm{g} / \mathrm{m}^{2}\right)$ & $28 \pm 5$ & $28 \pm 5$ & 0.458 \\
\hline Systolic blood pressure (mmHg) & $136 \pm 18$ & $137 \pm 18$ & 0.615 \\
\hline Diastolic blood pressure $(\mathrm{mmHg})$ & $80 \pm 9$ & $82 \pm 10$ & 0.288 \\
\hline $\begin{array}{l}\text { Antithrombotic drug at } \\
\text { baseline, } \%\end{array}$ & 99 & 99 & 0.637 \\
\hline - VKA at baseline, $\%$ & 69 & 82 & 0.032 \\
\hline - Aspirin at baseline, $\%$ & 32 & 18 & 0.023 \\
\hline \multicolumn{4}{|l|}{ Echocardiography } \\
\hline AF during TEE, $\%$ & 25 & 54 & $<0.001$ \\
\hline Left atrial diameter (mm) & $41 \pm 11$ & $42 \pm 12$ & 0.389 \\
\hline Left ventricular ejection fraction (\%) & $59 \pm 8$ & $56 \pm 8$ & 0.016 \\
\hline Left ventricular mass $\left(\mathrm{g} / \mathrm{m}^{2}\right)$ & $124 \pm 83$ & $130 \pm 102$ & 0.571 \\
\hline End-diastolic septum width (mm) & $9.6 \pm 2.6$ & $10.1 \pm 3.2$ & 0.204 \\
\hline End-diastolic posterior wall width (mm) & $9.1 \pm 2.4$ & $9.5 \pm 2.6$ & 0.211 \\
\hline Mitral incompetence, $\%$ & 55 & 70 & 0.038 \\
\hline
\end{tabular}

Data are expressed as mean \pm SD unless otherwise specified $A F$ atrial fibrillation, $V K A$ vita$\min \mathrm{K}$ antagonists, TEE transoesophageal echocardiography presence of SEC and LAA flow velocities, or complex plaques since it does not alter the underlying haemodynamic mechanism [14-16], nor aortic plaque anatomy and complexity [17], respectively. On the other hand, continued VKA therapy may have precluded finding thrombi on TEE. However, we reasoned that the incidence of thrombi after stopping VKA in patients randomised to aspirin would be low, especially since high LAA flow velocities and absence of SEC virtually exclude thrombus $[15,18]$. In this respect, of all three echocardiographic atrial thrombosis markers, LAA flow velocity measurement is the most robust concerning sensitivity and specificity for thrombosis risk $[8,19]$. Therefore, to prevent unjust randomisation as much as possible we used a higher than usual cut off of LAA flow velocity of $25 \mathrm{~cm} / \mathrm{s}$. Notwithstanding the above, temporary discontinuation of stable VKA treatment for e.g. 4 weeks to assess stroke risk markers with TEE off VKA may seem feasible since most patients eligible for the

TEE transoesophageal echocardiography 
TIARA approach have a relatively low stroke risk. Although the stroke risk during 4 weeks of interrupted VKA is low, we did not feel justified to do so, especially since the guidelines advise keeping discontinuation of VKA (e.g. to perform elective surgery) as short as possible.

The overall mean $\mathrm{CHADS}_{2}$ score was fairly low and median $\mathrm{CHADS}_{2}$ score was only 1 , mainly because we did not consider patients with a previous stroke or heart failure. In contrast, patients included in RACE [20] and AFFIRM [21] were older and were presumably in a higher $\mathrm{CHADS}_{2}$ risk category mostly due to previous stroke and heart failure, although hypertension was less frequent. Obviously, the patients enrolled in the ACTIVE trial [22] and RELY study [23] had higher $\mathrm{CHADS}_{2}$ scores because patients at higher risk for thromboembolism were included, whereas the TIARA pilot study intended to focus on patients with an intermediate risk, i.e. a lower $\mathrm{CHADS}_{2}$ score.

Patients who were excluded from randomisation (nonrandomised observational group) because of TEE abnormalities had, expectedly, a higher mean $\mathrm{CHADS}_{2}$ score than randomised patients, mainly due to higher average age and higher prevalence of diabetes. Nevertheless, the median $\mathrm{CHADS}_{2}$ score is 1 in both randomised and non-randomised groups. We can conclude that the average patient randomised in the TIARA pilot study was the younger patient with wellcontrolled hypertension and paroxysmal AF without other risk factors of the $\mathrm{CHADS}_{2}$ risk score (i.e. heart failure, previous stroke or TIA, diabetes, and older age). The relatively high number of women included in the TIARA study is remarkable, considering that women are generally underrepresented in clinical trials.

In the non-randomised observational group, ten thrombi were found (3.3\% of patients); eight of these patients were on VKA and two on aspirin. The number of thrombi as well as stroke risk category in our study is comparable with several recent studies showing prevalences of thrombi between $0.6 \%$ and 3.6\% [24-26]. Remarkably, also three patients (0.97\%) with a $\mathrm{CHADS}_{2}$ score of 0 had at least one TEE stroke risk factor. Scherr et al. also report a prevalence of $0.3 \%$ thrombi in patients with a $\mathrm{CHADS}_{2}$ score of 0 [25]. This underscores our hypothesis that current risk stratification schemeswhich are based merely on clinical characteristics (such as the $\mathrm{CHADS}_{2}$ score) - are unable to detect all patients with a high risk of stroke with certainty. Surprisingly, three patients had no thromboembolic markers but other abnormalities with an indication for treatment with VKA. These patients were asymptomatic beforehand, and they were subsequently treated with VKA and followed in de observational group. To our knowledge, this is the first study to show that routine TEE yields unexpected findings in AF patients, leading to a change of antithrombotic treatment.

Performing TEE in AF patients with a low to average thromboembolism risk $\left(\mathrm{CHADS}_{2}\right.$ score of 0 and 1$)$ is useful since it may enhance aspirin or no antithrombotic treatment in some of these patients which may reduce avoidable bleeding. In addition, in patients considered at low risk, TEE may detect high-risk features warranting VKA therapy, protecting these patients from unexpected strokes. In this respect it is noteworthy that out of 310 patients with an average $\mathrm{CHADS}_{2}$ risk score of 1,63 patients had an abnormal TEE (20.3\%) necessitating continuation of or switch to VKA therapy. Most noteworthy in this respect were the three patients with a CHADS 2 risk score of 0 who appeared to have thrombogenic features on TEE.

\section{Conclusions}

In summary, the TIARA pilot study showed that a TEEbased risk stratification in patients with atrial fibrillation and a moderate risk for stroke is feasible. Decreasing the threats and inconvenience of VKA is a desirable goal. Identifying patients in whom VKA (or other antithrombotic drugs) can be avoided may reduce bleeding and improve quality of life without an increase in stroke complications or costs of care. Application of a new echo-guided antithrombotic strategy may help to reduce bleeding whilst stroke prevention is maintained. If so, this study may significantly change daily clinical practice of antithrombotic management of atrial fibrillation. These results may form the basis for a larger multi-centre study.

Open Access This article is distributed under the terms of the Creative Commons Attribution Noncommercial License which permits any noncommercial use, distribution, and reproduction in any medium, provided the original author(s) and source are credited.

\section{Appendix}

\section{Principal Investigator}

H.J.G.M. Crijns.

\section{Steering Committee}

H.J.G.M. Crijns (chairman), T. Dinh, L.H.B. Baur, R. Pisters, O. Kamp, M.H. Prins, F.W.A. Verheugt.

\section{Central Independent Adjudication Committee}

C. de Zwaan (Department of Cardiology, chairman), M. Limburg (Department of Neurology), H. ten Cate (Department of Hematology), all Maastricht University Medical Center.

\section{Participating centres and persons; number in parenthe- ses indicates numbers of patients enrolled (all the Netherlands)}

Maastricht University Medical Center, Maastricht- $\mathrm{T}$. Dinh, H. Crijns, R. Pisters (133); Atrium Medical Center, 
Heerlen-L. Baur (121); VU Medical Center, AmsterdamO. Kamp (22); Rijnstate Hospital, Arnhem-J. Lindeboom (7); VieCuri Medical Center, Venlo_-F. Heesen (6); Radboud University Nijmegen Medical Center, Nijmegen_-J. Smeets (4); Twenteborg Hospital, Almelo-H. Idzerda (5); Jeroen Bosch Hospital, 's-Hertogenbosch-M. van Eck, H. Haerkens-Arends (4); Diakonessenhuis, Meppel-K. Thomas, H. Hoogslag (4); Amphia Hospital, Breda-M. Baselier, M. Alings, T. Simmers (4), all the Netherlands.

\section{Statistical analysis and data processing}

T. Dinh (Study Coordinator), M.H. Prins (Head Department of Clinical Epidemiology and Medical Technology Assessment), Center for Data and Information Management (MEMIC, Faculty of Health, Medicine and Life Sciences, Maastricht University).

\section{CardioResearch Maastricht University Medical Center monitoring activities}

I. Partouns-Hendriks, I. Ummels, L. Muis-Molin.

\section{Funding}

The TIARA pilot study was supported by grants of the Interuniversity Cardiology Institute of the Netherlands, the Maastricht University Medical Centre, CARIM Research Institute Maastricht, and an unrestricted grant from Astra Zeneca, the Netherlands.

\section{Definitions of primary endpoint events}

Stroke was subdivided into ischaemic stroke (including lacunar stroke), haemorrhagic stroke or undefined stroke. Each ischaemic stroke was further subdivided into cardioembolic, large-artery atherosclerotic stroke, lacunar stroke (i.e. small-artery occlusion) or mixed/undefined/other.

Death was divided into cardiovascular death (due to myocardial infarction, heart failure, documented stroke, procedure-related death, due to other cardiovascular causes) or non-cardiovascular death (related to cancer, primary infectious disease, respiratory disease, suicide, accident or other).

Major bleeding was defined as clinically overt and (a) associated with a fall in haemoglobin of $1.25 \mathrm{mmol} / 1$ or more, or (b) leading to a transfusion of 2 or more units of packed red blood cells or whole blood, or (c) bleeding in a critical organ, i.e. intracranial, retroperitoneal, pericardial, or (d) contributing to death. All other relevant bleeding was classified as a non-major bleeding.

\section{References}

1. Wolf PA, Abbott RD, Kannel WB. Atrial fibrillation as an independent risk factor for stroke: the Framingham Study. Stroke. 1991;22(8):983-8.
2. Wolf PA, Abbott RD, Kannel WB. Atrial fibrillation: a major contributor to stroke in the elderly. The Framingham Study. Arch Intern Med. 1987;147(9):1561-4.

3. Risk factors for stroke and efficacy of antithrombotic therapy in atrial fibrillation. Analysis of pooled data from five randomized controlled trials. Arch Intern Med. 1994;154(13):1449-57.

4. Hart RG, Pearce LA, Aguilar MI. Meta-analysis: antithrombotic therapy to prevent stroke in patients who have nonvalvular atrial fibrillation. Ann Intern Med. 2007;146(12):857-67.

5. Fuster V, Ryden LE, Asinger RW, et al. ACC/AHA/ESC guidelines for the management of patients with atrial fibrillation: executive summary. A Report of the American College of Cardiology/American Heart Association Task Force on Practice Guidelines and the European Society of Cardiology Committee for Practice Guidelines and Policy Conferences (Committee to Develop Guidelines for the Management of Patients With Atrial Fibrillation): developed in Collaboration With the North American Society of Pacing and Electrophysiology. J Am Coll Cardiol. 2001;38(4):1231-66.

6. Zabalgoitia M, Halperin JL, Pearce LA, et al. Transesophageal echocardiographic correlates of clinical risk of thromboembolism in nonvalvular atrial fibrillation. Stroke Prevention in Atrial Fibrillation III Investigators. J Am Coll Cardiol. 1998;31(7):1622-6.

7. Blackshear JL, Pearce LA, Hart RG, et al. Aortic plaque in atrial fibrillation: prevalence, predictors, and thromboembolic implications. Stroke. 1999;30(4):834-40.

8. Kamp O, Verhorst PM, Welling RC, et al. Importance of left atrial appendage flow as a predictor of thromboembolic events in patients with atrial fibrillation. Eur Heart J. 1999;20(13):979-85.

9. Shanewise JS, Cheung AT, Aronson S, et al. ASE/SCA guidelines for performing a comprehensive intraoperative multiplane transesophageal echocardiography examination: recommendations of the American Society of Echocardiography Council for Intraoperative Echocardiography and the Society of Cardiovascular Anesthesiologists Task Force for Certification in Perioperative Transesophageal Echocardiography. J Am Soc Echocardiogr. 1999;12(10):884-900.

10. Manning WJ, Weintraub RM, Waksmonski CA, et al. Accuracy of transesophageal echocardiography for identifying left atrial thrombi. A prospective, intraoperative study. Ann Intern Med. 1995;123(11):817-22.

11. Verhorst PM, Kamp O, Visser CA, et al. Left atrial appendage flow velocity assessment using transesophageal echocardiography in nonrheumatic atrial fibrillation and systemic embolism. Am J Cardiol. 1993;71(2):192-6.

12. Stoddard MF, Liddell NE, Longaker RA, et al. Transesophageal echocardiography: normal variants and mimickers. Am Heart J. 1992;124(6): 1587-98.

13. Roldan CA, Shively BK, Crawford MH. Valve excrescences: prevalence, evolution and risk for cardioembolism. J Am Coll Cardiol. 1997;30(5):1308-14.

14. Fatkin D, Loupas T, Low J, et al. Inhibition of red cell aggregation prevents spontaneous echocardiographic contrast formation in human blood. Circulation. 1997;96(3):889-96.

15. Fatkin D, Kelly RP, Feneley MP. Relations between left atrial appendage blood flow velocity, spontaneous echocardiographic contrast and thromboembolic risk in vivo. J Am Coll Cardiol. 1994;23(4):961-9.

16. Tsai LM, Chen JH, Lin LJ, et al. Natural history of left atrial spontaneous echo contrast in nonrheumatic atrial fibrillation. Am J Cardiol. 1997;80(7):897-900.

17. Thenappan T, Ali Raza J, Movahed A. Aortic atheromas: current concepts and controversies-a review of the literature. Echocardiography. 2008;25(2):198-207.

18. Handke M, Harloff A, Hetzel A, et al. Left atrial appendage flow velocity as a quantitative surrogate parameter for thromboembolic risk: determinants and relationship to spontaneous echocontrast 
and thrombus formation-a transesophageal echocardiographic study in 500 patients with cerebral ischemia. J Am Soc Echocardiogr. 2005;18(12):1366-72.

19. Dawn B, Varma J, Singh P, et al. Cardiovascular death in patients with atrial fibrillation is better predicted by left atrial thrombus and spontaneous echocardiographic contrast as compared with clinical parameters. J Am Soc Echocardiogr. 2005;18(3):199-205.

20. Van Gelder IC, Hagens VE, Bosker HA, et al. A comparison of rate control and rhythm control in patients with recurrent persistent atrial fibrillation. N Engl J Med. 2002;347(23): 1834-40.

21. Baseline characteristics of patients with atrial fibrillation: the AFFIRM Study. Am Heart J. 2002;143(6):991-1001.

22. Connolly SJ, Pogue J, Hart RG, et al. Effect of clopidogrel added to aspirin in patients with atrial fibrillation. $\mathrm{N}$ Engl $\mathrm{J}$ Med. 2009;360(20):2066-78.
23. Connolly SJ, Ezekowitz MD, Yusuf S, et al. Dabigatran versus Warfarin in patients with atrial fibrillation. N Engl J Med. 2009.

24. Puwanant S, Varr BC, Shrestha K, et al. Role of the CHADS2 score in the evaluation of thromboembolic risk in patients with atrial fibrillation undergoing transesophageal echocardiography before pulmonary vein isolation. J Am Coll Cardiol. 2009;54 (22):2032-9.

25. Scherr D, Dalal D, Cheema A, et al. Long- and short-term temporal stability of complex fractionated atrial electrograms in human left atrium during atrial fibrillation. J Cardiovasc Electrophysiol. 2009;20(1):13-21.

26. Wallace TW, Atwater BD, Daubert JP, et al. Prevalence and clinical characteristics associated with left atrial appendage thrombus in fully anticoagulated patients undergoing catheterdirected atrial fibrillation ablation. J Cardiovasc Electrophysiol. 2010;21(8):849-52 